\title{
A Cylindrical Model for Studying Subendocardial \\ Ischaemia in the Left Ventricle
}

\author{
Peter R. Johnston
}

School of Science,

Griffith University,

Nathan,

Queensland,

Australia, 4111,

Phone: 61-7-3875-7748,

Fax: 61-7-3875-7656,

E-mail: P.Johnston@griffith.edu.au

July 14, 2003 


\begin{abstract}
In this paper a mathematical model of a left ventricle with a cylindrical geometry is presented with the aim of gaining a better understanding of the relationship between subendocardial ischaemia and ST depression. The model is formulated as an infinite cylinder and takes into account the full bidomain nature of cardiac tissue, as well as fibre rotation. A detailed solution method (based on Fourier series, Fourier transforms and a one dimensional finite difference scheme) for the governing equations for electric potential in the tissue and the blood is also presented.

The model presented is used to study the effect increasing subendocardial ischaemia has on the epicardial potential distribution as well as the effects of changing the bidomain conductivity values. The epicardial potential distributions obtained with this cylindrical geometry are compared with results obtained using a previously published slab model. Results of the simulations presented show that the morphologies of the epicardial potential distributions are similar between the two geometries, with the main difference being that the cylindrical model predicts slightly higher potentials.
\end{abstract}

Keywords: Bidomain Model, Anisotropy, ST Depression, Cylindrical Geometry, Subendocardial Ischaemia. 


\section{Introduction}

For many years, electrocardiographic ST segment depression has been used as an indicator of cardiac ischaemia [1]. However, there is still some degree of controversy regarding the mechanisms responsible for this phenomenon [2]. In an effort to further understanding of this relationship, a mathematical model for ST segment changes due to subendocardial ischaemia has been introduced for a slab of cardiac tissue [3]. The model utilises the bidomain representation of cardiac tissue [4] and the conductivity data of Clerc [5] to study the effect of increasing subendocardial ischaemia on epicardial potential distributions. Recently, the same model was employed to study the effect different sets of published conductivity values have on the epicardial potential distributions [6].

A limitation of the slab model is the inherent assumption that it can represent only a small region of the myocardium. That is to say, it is assumed that the region of ventricular wall which is being considered is perfectly flat. Clearly, this can only be true for small regions of ventricular wall, if it is true at all. Consequently, another limitation is the assumption that there is an infinite blood mass attached to the slab of tissue.

To overcome these limitations, this paper presents a model of the left ventricle in the form of an infinite cylinder. The advantage of this approach is that the curvature of the outside wall is introduced, as is the inclusion of a "smaller" blood mass. Here, "smaller" should be interpreted as meaning that there is a finite extent to the blood mass in at least one direction.

Another advantage of this approach is that solution of the governing equations for the electric potential in the tissue and the blood can be achieved with only minimal reliance on numerical methods. Hence, this approach can serve as a test solution for full three dimensional numerical solution methodologies for the same problem. Solution of the governing equations is achieved using Fourier series and Fourier transform techniques, along with a one dimensional 
finite difference scheme.

The object of this paper is to present the detailed solution method for the governing equations within the cardiac tissue and the blood mass. Then, the solution is used to compare this cylindrical model with the slab model, in terms of epicardial surface potential distributions, as the degree of subendocardial ischaemia is increased to full thickness. Finally, the effect on the epicardial potential distribution of different bidomain conductivity values is considered for the cylindrical geometry and then compared to the slab model.

\section{Description of the Model}

\subsection{Governing Equations}

It will be assumed that the ventricular muscle can be approximated by an annulus, infinite in the $\mathrm{z}$ direction and extending from $r=r_{a}$ (endocardium) to $r=r_{b}$ (epicardium) in the radial direction. The interior of the annular region is assumed to be filled with blood and the outer surface of the cylinder is insulated. Figure 1 shows a cross section through the cylinder at $z=0$. The cross hatched area indicates the region of subendocardial ischaemia, which will be discussed shortly.

Effects of both the intracellular and extracellular regions of the cardiac tissue are included through the use of the bidomain model $[4,7,8,9]$ for cardiac tissue. In order to solve the two bidomain equations governing the intracellular potentials, $\phi_{i}$, and the extracellular potentials, $\phi_{e}$, the transmembrane potential $\phi_{m}=\phi_{i}-\phi_{e}$ is introduced. It can be shown that $[7,8,9]$ the governing equation for $\phi_{e}$ is

$$
\nabla \cdot\left(\mathbf{M}_{i}+\mathbf{M}_{e}\right) \nabla \phi_{e}=-\nabla \cdot \mathbf{M}_{i} \nabla \phi_{m}
$$

where $M_{e}$ and $M_{i}$ are conductivity tensors reflecting the anisotropy of the cardiac tissue and $\nabla$ 
is the gradient operator in a cylindrical coordinate system. Here, $\phi_{e}$, can be determined from a knowledge of the transmembrane potential distribution which is known from individual cell action potentials.

Finally, in the blood, being a source free region, the electric potential, $\phi_{b}$, is governed by Laplace's equation

$$
\nabla^{2} \phi_{b}=0
$$

again in cylindrical coordinates.

\subsection{Conductivity Tensor}

Cardiac tissue is an electrically anisotropic structure, consisting of sheets of parallel strands of cells, where it is much easier for current to flow along the fibres than across them. Therefore, four conductivity values are required to fully describe the conductivity in the intracellular and extracellular spaces: $\sigma_{l}^{i}, \sigma_{t}^{i}, \sigma_{l}^{e}, \sigma_{t}^{e}$, where the superscripts $i$ and $e$ refer to intracellular and extracellular domains, respectively, and the subscripts $l$ and $t$ refer to longitudinal and transverse directions, respectively. Here, longitudinal direction means along the direction of the fibres and transverse means across the fibres, perpendicular to the longitudinal direction within a sheet. In this annular model, it is assumed that the sheets of fibres wrap around the cylindrical core and that the radial $(r)$ direction is perpendicular to these sheets. Hence, it is assumed that the conductivity in the $r$ direction is the same as in the transverse direction. This formulation, thus, ignores the secondary effects of sheet structure.

Many investigators have observed that the main fibre direction rotates in a counterclockwise direction as one moves from the epicardium to the endocardium. For the left ventricle, rotations in fibre direction have been reported in the range of $103 \pm 21^{\circ}$ [10] up to $180^{\circ}$ [11]. It is also common to assume that the rotation varies linearly with depth [12]. Therefore, if the fibres on 
the epicardium are initially offset from the $z$-axis by an angle of $\psi_{0}$, then the direction of the fibres at a position $r$ in the ventricular muscle $\left(r_{a} \leq r \leq r_{b}\right)$ is given by

$$
g(r)=\psi_{0}+\psi\left(\frac{r_{b}-r}{r_{b}-r_{a}}\right)
$$

where $\psi$ is the total rotation in fibre direction from the epicardium to the endocardium.

Given the above discussion, it follows that the conductivity tensors in equation (1) can be represented as $3 \times 3$ matrices of the form

$$
\mathbf{M}_{n}(z, \theta, r)=\left(\begin{array}{ccc}
\left(\sigma_{l}^{n}-\sigma_{t}^{n}\right) c^{2}+\sigma_{t}^{n} & \left(\sigma_{l}^{n}-\sigma_{t}^{n}\right) c s & 0 \\
\left(\sigma_{l}^{n}-\sigma_{t}^{n}\right) c s & \left(\sigma_{l}^{n}-\sigma_{t}^{n}\right) s^{2}+\sigma_{t}^{n} & 0 \\
0 & 0 & \sigma_{t}^{n}
\end{array}\right)
$$

where $n=i$ or $e$ (for intracellular or extracellular), $c=\cos (g(r))$ and $s=\sin (g(r))$.

Finally, it is assumed that the imbrication angle (the angle of inclination of the fibres relative to the epicardial surface) is zero. This is a reasonably common assumption [12] and is justified in this situation because the model presented here approximates the ventricular wall, away from apex and base, where the imbrication angle is less than $5^{\circ}[13]$.

\subsection{Region of Subendocardial Ischaemia}

It will be assumed that the ischaemic tissue occupies a finite region within the ventricular muscle, described by

$$
I=\left\{(z, \theta, r) \mid-a_{z} \leq z \leq a_{z},-a_{\theta} \leq \theta \leq a_{\theta}, r_{a} \leq r \leq r_{a}+a_{r}\right\}
$$

Here $a_{z}$ represents the half length of the ischaemic region in the $z$ direction, and $a_{\theta}$ is the angle subtended by the ischaemic region at the $z$-axis (see Figure 1). In the radial direction, the ischaemic region begins at the endocardium but does not extend all the way to the epicardium, just to some distance $a_{r}$ into the ventricular wall. It will be assumed that there is a smooth 
sigmoidal transition between ischaemic and normal tissue across the ischaemic boundary, where $a_{z}, a_{\theta}$ and $r_{a}+a_{r}$ represent the midpoints of the ischaemic boundaries in the $z, \theta$ and $r$ directions, respectively.

In the ischaemic region the cells have been damaged due to lack of oxygen, the result of which is to reduce the plateau transmembrane potential compared to that of the normal cells. This ischaemic region can be represented in an analytic fashion as a product of the transmembrane potential distributions in the three principal coordinate directions $[7,3]$

$$
\phi_{m}(z, \theta, r)=\Delta \phi_{p} \Psi(z) \Psi(\theta) \Psi\left(r+r_{a}\right) .
$$

Here, $\Delta \phi_{p}$ is the difference in plateau potentials between normal and ischaemic tissue. In any particular direction, $t$, the shape function $\Psi(t)$ is defined by

$$
\Psi(t)= \begin{cases}\frac{1-e^{-a_{t} / \lambda_{t}} \cosh t / \lambda_{t}}{1-e^{-a_{t} / \lambda_{t}}} & |t| \leq a_{t} \\ \frac{e^{-|t| / \lambda_{t}} \sinh a_{t} / \lambda_{t}}{1-e^{-a_{t} / \lambda_{t}}} & |t|>a_{t}\end{cases}
$$

where $t$ is $z, \theta$ or $r$. The parameters, $\lambda_{t}(t=z, \theta, r)$ govern the width of the ischaemic boundary and, as mentioned above, $a_{t}(t=z, \theta, r)$ is the midpoint of the ischaemic boundary. Note that in equation (6), the argument of the shape function in the $r$ direction is $r+r_{a}$ to create the ischaemic region outwards from the endocardium at $r=r_{a}$.

\subsection{Boundary Conditions}

A set of boundary conditions is required to solve the governing equations (1) and (2). Since the cylinder is infinitely long in the $z$ direction, it is assumed that $\phi_{e}=\phi_{b}=0$ as $z \rightarrow \pm \infty$. Also, as the whole model is insulated on the epicardium, the outer surface boundary condition is that

$$
\text { at } r=r_{b} ; \frac{\partial \phi_{e}}{\partial r}=0 \text {. }
$$


This insulation type boundary condition is used so that comparisons can be made with previously published simulation $[3,6]$ and experimental [14] studies in which the heart was insulated. The method presented here could be extended to consider the "heart" inside a bath, representing the torso, which is then insulated.

At the interface between the tissue and the blood, there is continuity of potential and current;

$$
\text { at } r=r_{a} ; \phi_{e}=\phi_{b} \quad \text { and } \quad \sigma_{b} \frac{\partial \phi_{b}}{\partial r}=\sigma_{t}^{e} \frac{\partial \phi_{e}}{\partial r}
$$

where $\sigma_{b}$ is the conductivity of blood. The form of the transmembrane potential distribution, equation (7) used in this model does not exactly fit with the above boundary conditions, but can be shown to be a good approximation. From [15], the assumption of a constant transmembrane potential distribution near the boundary is acceptable when the width of the tissue region (in this case $r_{b}-r_{a}$ ) is much larger than the length constant in the radial direction. Based on the assumption that the cells have a radius of approximately $10 \mu \mathrm{m}$, with membrane resistance times unit area of $0.91 \Omega \mathrm{m}^{2}$ and a volume fraction of 0.7 [15] then the length constants for each set of conductivities given in Table 1 are less than $0.5 \mathrm{~mm}$. As will be seen later $r_{b}-r_{a}=1 \mathrm{~cm}$, which is much larger than the length constant and so the approximation used here is reasonable.

\section{Solution Method}

The model proposed is three dimensional in a cylindrical coordinate system and the governing equation (1) can be written explicitly as

$$
\begin{aligned}
\frac{M_{i}^{33}+M_{e}^{33}}{r} \frac{\partial}{\partial r}\left(r \frac{\partial \phi_{e}}{\partial r}\right) & +\frac{M_{i}^{22}+M_{e}^{22}}{r^{2}} \frac{\partial^{2} \phi_{e}}{\partial \theta^{2}}+\frac{2\left(M_{i}^{12}+M_{e}^{12}\right)}{r} \frac{\partial^{2} \phi_{e}}{\partial \theta \partial z}+\left(M_{i}^{11}+M_{e}^{11}\right) \frac{\partial^{2} \phi_{e}}{\partial z^{2}} \\
& =-\frac{M_{i}^{33}}{r} \frac{\partial}{\partial r}\left(r \frac{\partial \phi_{m}}{\partial r}\right)-\frac{M_{i}^{22}}{r^{2}} \frac{\partial^{2} \phi_{m}}{\partial \theta^{2}}-\frac{2 M_{i}^{12}}{r} \frac{\partial^{2} \phi_{m}}{\partial \theta \partial z}-M_{i}^{11} \frac{\partial^{2} \phi_{m}}{\partial z^{2}}
\end{aligned}
$$

where $M_{n}^{m j}$ represents the elements of the conductivity tensor matrices $\mathbf{M}_{n}(\mathrm{n}=\mathrm{i}, \mathrm{e})$. Recall that $M_{n}^{11}, M_{n}^{12}$ and $M_{n}^{22}(n=i, e)$ are functions of $r$, via the fibre rotation and $M_{n}^{33}(n=i, e)$ 
are constant.

Similarly, the Laplace's equation in the blood region is given by

$$
\frac{1}{r} \frac{\partial}{\partial r}\left(r \frac{\partial \phi_{b}}{\partial r}\right)+\frac{1}{r^{2}} \frac{\partial^{2} \phi_{b}}{\partial \theta^{2}}+\frac{\partial^{2} \phi_{b}}{\partial z^{2}}=0
$$

There are several methods available to solve the above equations. Numerical solution techniques such as the finite difference method or the finite element method are ideally suited to such problems, but, here an analytical-numerical approach is adopted which keeps the numerical techniques to a minimum. One reason for doing this is to provide a solution which can be used to check purely numerical solution methods.

\subsection{Exploiting Periodicity of the Solution}

The first step is to exploit the periodic nature of the functions $\phi_{e}, \phi_{b}$ and $\phi_{m}$ in the $\theta$ direction and expand each in terms of a Fourier series:

$$
\phi_{K}=\frac{1}{2} A_{0}^{K}(r, z)+\sum_{k=1}^{\infty} A_{k}^{K}(r, z) \cos k \theta+B_{k}^{K}(r, z) \sin k \theta
$$

where $K=e, b$ or $m$. It is now necessary to find the coefficient functions $A_{k}^{K}(r, z)(k=0,1, \ldots)$ and $B_{k}^{K}(r, z)(k=1,2, \ldots)$. To this end, substitute equation (12) into equations (10) and (11) and equate coefficients of $\cos k \theta$ and $\sin k \theta$. This gives, firstly for $k=0$

$$
\begin{aligned}
\frac{M_{i}^{33}+M_{e}^{33}}{r} \frac{\partial}{\partial r}\left(r \frac{\partial A_{0}^{e}(r, z)}{\partial r}\right) & +\left(M_{i}^{11}+M_{e}^{11}\right) \frac{\partial^{2} A_{0}^{e}(r, z)}{\partial z^{2}} \\
& =-\frac{M_{i}^{33}}{r} \frac{\partial}{\partial r}\left(r \frac{\partial A_{0}^{m}(r, z)}{\partial r}\right)-M_{i}^{11} \frac{\partial^{2} A_{0}^{m}(r, z)}{\partial z^{2}}
\end{aligned}
$$

and

$$
\frac{1}{r} \frac{\partial}{\partial r}\left(r \frac{\partial A_{0}^{b}(r, z)}{\partial r}\right)+\frac{\partial^{2} A_{0}^{b}(r, z)}{\partial z^{2}}=0
$$


For arbitrary $k$, equating the coefficients of $\cos k \theta$ gives

$$
\begin{gathered}
\frac{M_{i}^{33}+M_{e}^{33}}{r} \frac{\partial}{\partial r}\left(r \frac{\partial A_{k}^{e}(r, z)}{\partial r}\right)-\frac{M_{i}^{22}+M_{e}^{22}}{r^{2}} k^{2} A_{k}^{e}(r, z)+2 k \frac{M_{i}^{12}+M_{e}^{12}}{r} \frac{\partial B_{k}^{e}(r, z)}{\partial z} \\
+\left(M_{i}^{11}+M_{e}^{11}\right) \frac{\partial^{2} A_{k}^{e}(r, z)}{\partial z^{2}} \\
=-\frac{M_{i}^{33}}{r} \frac{\partial}{\partial r}\left(r \frac{\partial A_{k}^{m}(r, z)}{\partial r}\right)+\frac{M_{i}^{22}}{r^{2}} k^{2} A_{k}^{m}(r, z)-2 k \frac{M_{i}^{12}}{r} \frac{\partial B_{k}^{m}(r, z)}{\partial z} \\
-M_{i}^{11} \frac{\partial^{2} A_{k}^{m}(r, z)}{\partial z^{2}}
\end{gathered}
$$

and equating the coefficients of $\sin k \theta$ gives

$$
\begin{gathered}
\frac{M_{i}^{33}+M_{e}^{33}}{r} \frac{\partial}{\partial r}\left(r \frac{\partial B_{k}^{e}(r, z)}{\partial r}\right)-\frac{M_{i}^{22}+M_{e}^{22}}{r^{2}} k^{2} B_{k}^{e}(r, z)-2 k \frac{M_{i}^{12}+M_{e}^{12}}{r} \frac{\partial A_{k}^{e}(r, z)}{\partial z} \\
+\left(M_{i}^{11}+M_{e}^{11}\right) \frac{\partial^{2} B_{k}^{e}(r, z)}{\partial z^{2}} \\
=-\frac{M_{i}^{33}}{r} \frac{\partial}{\partial r}\left(r \frac{\partial B_{k}^{m}(r, z)}{\partial r}\right)+\frac{M_{i}^{22}}{r^{2}} k^{2} B_{k}^{m}(r, z)+2 k \frac{M_{i}^{12}}{r} \frac{\partial A_{k}^{m}(r, z)}{\partial z} \\
-M_{i}^{11} \frac{\partial^{2} B_{k}^{m}(r, z)}{\partial z^{2}}
\end{gathered}
$$

For the blood region the two equations for $A_{k}^{b}(r, z)$ and $B_{k}^{b}(r, z)$ are

$$
\frac{1}{r} \frac{\partial}{\partial r}\left(r \frac{\partial A_{k}^{b}(r, z)}{\partial r}\right)-\frac{k^{2}}{r^{2}} A_{k}^{b}(r, z)+\frac{\partial^{2} A_{k}^{b}(r, z)}{\partial z^{2}}=0
$$

and

$$
\frac{1}{r} \frac{\partial}{\partial r}\left(r \frac{\partial B_{k}^{b}(r, z)}{\partial r}\right)-\frac{k^{2}}{r^{2}} B_{k}^{b}(r, z)+\frac{\partial^{2} B_{k}^{b}(r, z)}{\partial z^{2}}=0
$$

The boundary conditions for these unknown coefficient functions are

$$
\begin{gathered}
\text { at } r=0: \frac{\partial A_{k}^{b}(r, z)}{\partial r}=\frac{\partial B_{k}^{b}(r, z)}{\partial r}=0 \\
\text { at } r=r_{a}: A_{k}^{e}(r, z)=A_{k}^{b}(r, z), B_{k}^{e}(r, z)=B_{k}^{b}(r, z), \\
\quad \sigma_{b} \frac{\partial A_{k}^{b}(r, z)}{\partial r}=\sigma_{t}^{e} \frac{\partial A_{k}^{e}(r, z)}{\partial r}, \sigma_{b} \frac{\partial B_{k}^{b}(r, z)}{\partial r}=\sigma_{t}^{e} \frac{\partial B_{k}^{e}(r, z)}{\partial r} \\
\text { at } r=r_{b}: \frac{\partial A_{k}^{e}(r, z)}{\partial r}=\frac{\partial B_{k}^{e}(r, z)}{\partial r}=0 \\
\text { as } z \rightarrow \pm \infty: A_{k}^{e}(r, z)=A_{k}^{b}(r, z)=B_{k}^{e}(r, z)=B_{k}^{b}(r, z)=0
\end{gathered}
$$




\subsection{Reduction to Ordinary Differential Equations}

The above partial differential equations can be further reduced to ordinary differential equations by introducing a Fourier transform in the $z$-direction. Define

$$
C_{k l}^{K}(r)=\int_{-\infty}^{\infty} A_{k}^{K}(r, z) e^{-2 \pi i l z} d z
$$

and

$$
D_{k l}^{K}(r)=\int_{-\infty}^{\infty} B_{k}^{K}(r, z) e^{-2 \pi i l z} d z
$$

where $K=e, m$ or $b$. Applying the transformation (19) (for $l>0$ ) to equations (13) and (14) (ie $\mathrm{k}=0$ ) gives

$$
\begin{aligned}
\frac{M_{i}^{33}+M_{e}^{33}}{r} \frac{d}{d r}\left(r \frac{d C_{0 l}^{e}(r)}{d r}\right) & -4 \pi^{2} l^{2}\left(M_{i}^{11}+M_{e}^{11}\right) C_{0 l}^{e}(r) \\
& =-\frac{M_{i}^{33}}{r} \frac{d}{d r}\left(r \frac{d C_{0 l}^{m}(r)}{d r}\right)+4 \pi^{2} l^{2} M_{i}^{11} C_{0 l}^{m}(r)
\end{aligned}
$$

and

$$
\frac{1}{r} \frac{d}{d r}\left(r \frac{d C_{0 l}^{e}(r)}{d r}\right)-4 \pi^{2} l^{2} C_{0 l}^{e}(r)=0
$$

Next application of the Fourier transforms to equations (15) and (16) gives

$$
\begin{aligned}
\frac{M_{i}^{33}+M_{e}^{33}}{r} \frac{d}{d r}\left(r \frac{d C_{k l}^{e}(r)}{d r}\right) & -\left[\frac{M_{i}^{22}+M_{e}^{22}}{r^{2}} k^{2}+4 \pi^{2} l^{2}\left(M_{i}^{11}+M_{e}^{11}\right)\right] C_{k l}^{e}(r) \\
& +4 \pi i l k \frac{M_{i}^{12}+M_{e}^{12}}{r} D_{k l}^{e}(r) \\
= & -\frac{M_{i}^{33}}{r} \frac{d}{d r}\left(r \frac{d C_{k l}^{m}(r)}{d r}\right)+\left[\frac{M_{i}^{22}}{r^{2}} k^{2}+4 \pi^{2} l^{2} M_{i}^{11}\right] C_{k l}^{m}(r)
\end{aligned}
$$

and

$$
\begin{aligned}
\frac{M_{i}^{33}+M_{e}^{33}}{r} \frac{d}{d r}\left(r \frac{d D_{k l}^{e}(r)}{d r}\right)- & {\left[\frac{M_{i}^{22}+M_{e}^{22}}{r^{2}} k^{2}+4 \pi^{2} l^{2}\left(M_{i}^{11}+M_{e}^{11}\right)\right] D_{k l}^{e}(r) } \\
& -4 \pi i l k \frac{M_{i}^{12}+M_{e}^{12}}{r} C_{k l}^{e}(r) \\
= & 4 \pi i l k \frac{M_{i}^{12}}{r} C_{k l}^{m}(r)
\end{aligned}
$$

where the fact that $D_{k l}^{m}(r)=0$ has been used (this will be established later). Similar application of the Fourier transforms to equations (17) and (18) gives

$$
\frac{1}{r} \frac{d}{d r}\left(r \frac{d C_{k l}^{b}(r)}{d r}\right)-\left[\frac{k^{2}}{r^{2}}+4 \pi^{2} l^{2}\right] C_{k l}^{b}(r)=0
$$


and

$$
\frac{1}{r} \frac{d}{d r}\left(r \frac{d D_{k l}^{b}(r)}{d r}\right)-\left[\frac{k^{2}}{r^{2}}+4 \pi^{2} l^{2}\right] D_{k l}^{b}(r)=0
$$

Finally, applying the Fourier transform with $l=0$ to equations (13), (14), (15), (16), (17) and (18) gives

$$
\begin{gathered}
\frac{M_{i}^{33}+M_{e}^{33}}{r} \frac{d}{d r}\left(r \frac{d C_{00}^{e}(r)}{d r}\right)=-\frac{M_{i}^{33}}{r} \frac{d}{d r}\left(r \frac{d C_{00}^{m}(r)}{d r}\right) \\
\frac{1}{r} \frac{d}{d r}\left(r \frac{d C_{00}^{b}(r)}{d r}\right)=0 \\
\frac{M_{i}^{33}+M_{e}^{33}}{r} \frac{d}{d r}\left(r \frac{d C_{k 0}^{e}(r)}{d r}\right)-\frac{M_{i}^{22}+M_{e}^{22}}{r^{2}} k^{2} C_{k 0}^{e}(r) \\
=-\frac{M_{i}^{33}}{r} \frac{d}{d r}\left(r \frac{d C_{k 0}^{m}(r)}{d r}\right)+\frac{M_{i}^{22}}{r^{2}} k^{2} C_{k 0}^{m}(r) \\
\frac{M_{i}^{33}+M_{e}^{33}}{r} \frac{d}{d r}\left(r \frac{d D_{k 0}^{e}(r)}{d r}\right)-\frac{M_{i}^{22}+M_{e}^{22}}{r^{2}} k^{2} D_{k 0}^{e}(r)=0 \\
\frac{1}{r} \frac{d}{d r}\left(r \frac{d C_{k 0}^{b}(r)}{d r}\right)-\frac{k^{2}}{r^{2}} C_{k 0}^{b}(r)=0 \\
\frac{1}{r} \frac{d}{d r}\left(r \frac{d D_{k 0}^{b}(r)}{d r}\right)-\frac{k^{2}}{r^{2}} D_{k 0}^{b}(r)=0
\end{gathered}
$$

Application of the Fourier transform to the boundary conditions gives

$$
\begin{aligned}
& \text { at } r=0: \frac{d C_{k l}^{b}(r)}{d r}=\frac{d D_{k l}^{b}(r)}{d r}=0 \\
& \text { at } r=r_{a}: C_{k l}^{b}(r)=C_{k l}^{e}(r), D_{k l}^{b}(r)=D_{k l}^{e}(r), \\
& \qquad \sigma_{b} \frac{d C_{k l}^{b}(r)}{d r}=\sigma_{t}^{e} \frac{d C_{k l}^{e}(r)}{d r}, \sigma_{b} \frac{d D_{k l}^{b}(r)}{d r}=\sigma_{t}^{e} \frac{d D_{k l}^{e}(r)}{d r} \\
& \text { at } r=r_{b}: \frac{d C_{k l}^{e}(r)}{d r}=\frac{d D_{k l}^{e}(r)}{d r}=0
\end{aligned}
$$

These boundary conditions apply for all values of $l$, including $l=0$ and all appropriate values of $k$.

\subsection{Solution of the Ordinary Differential Equations}

Of the 12 ordinary differential equations which result from the initial governing equations, several can be solved analytically and will be considered first. The remainder can be solved numerically using a simple one dimensional finite difference scheme. 
Firstly, equation (22) is a modified Bessel equation of order zero and the solution is

$$
C_{0 l}^{b}(r)=a_{0}^{l} I_{0}(2 \pi l r)+b_{0}^{l} K_{0}(2 \pi l r) \quad 0 \leq r \leq r_{a}
$$

where $I_{0}$ and $K_{0}$ are zero order modified Bessel functions of the first and second kind, respectively. Applying the boundary condition at $r=0$ gives

$$
C_{0 l}^{b}(r)=a_{0}^{l} I_{0}(2 \pi l r) \quad 0 \leq r \leq r_{a}
$$

as $K_{0}(2 \pi l r)$ is unbounded as $r \rightarrow 0$. Equations (25) and (26) are also modified Bessel equations, this time of order $k$, so the solutions after application of the boundary conditions at $r=0$ are

$$
C_{k l}^{b}(r)=a_{k}^{l} I_{k}(2 \pi l r) \quad 0 \leq r \leq r_{a}
$$

and

$$
D_{k l}^{b}(r)=f_{k}^{l} I_{k}(2 \pi l r) \quad 0 \leq r \leq r_{a}
$$

Equations (27) and (28) can be solved by integrating twice with respect to $r$. Applying the appropriate boundary conditions gives the solutions

$$
C_{00}^{e}(r)=\frac{M_{i}^{33}}{M_{i}^{33}+M_{e}^{33}}\left[\left.r_{b} \frac{d C_{00}^{m}(r)}{d r}\right|_{r=r_{b}} \ln \frac{r}{r_{b}}+C_{00}^{m}\left(r_{a}\right)-C_{00}^{m}(r)\right]
$$

and

$$
C_{00}^{b}(r)=0
$$

Finally, equations (31) and (32) are Euler equations and so, after applying the boundary conditions at $r=0$, their solutions are

$$
C_{k 0}^{b}(r)=a_{k}^{0} r^{k}
$$

and

$$
D_{k 0}^{b}(r)=f_{k}^{0} r^{k}
$$


The remaining equations must be solved numerically, using a simple one dimensional finite difference scheme, as the coefficients $M_{n}^{11}, M_{n}^{12}$ and $M_{n}^{22}(n=i, e)$ are functions of $r$. Equations (21), (29) and (30) are independent equations and can be solved in isolation; however, equations (23) and (24) are coupled together and both contain imaginary components.

To simplify the discussion of the solutions of equations (23) and (24), introduce the following variables: $Y(r)=C_{k l}^{e}(r), Z(r)=D_{k l}^{e}(r), A=M_{i}^{33}+M_{e}^{33}$,

$$
\begin{aligned}
F(r) & =\frac{M_{i}^{22}+M_{e}^{22}}{r^{2}} k^{2}+4 \pi^{2} l^{2}\left(M_{i}^{11}+M_{e}^{11}\right) \\
G(r) & =4 \pi l k \frac{M_{i}^{12}+M_{e}^{12}}{r} \\
H_{1}(r) & =-\frac{M_{i}^{33}}{r} \frac{d}{d r}\left(r \frac{d C_{k l}^{m}(r)}{d r}\right)+\left[\frac{M_{i}^{22}}{r^{2}} k^{2}+4 \pi^{2} l^{2} M_{i}^{11}\right] C_{k l}^{m}(r) \\
H_{2}(r) & =4 \pi i l k \frac{M_{i}^{12}}{r} C_{k l}^{m}(r)
\end{aligned}
$$

then the equations become

$$
\begin{aligned}
& \frac{A}{r} \frac{d}{d r}\left(r \frac{d Y}{d r}\right)-F(r) Y+G(r) i Z=H_{1}(r) \\
& \frac{A}{r} \frac{d}{d r}\left(r \frac{d Z}{d r}\right)-F(r) Z-G(r) i Y=H_{2}(r) i
\end{aligned}
$$

Multiplying equation (42) by $i$ gives

$$
\frac{A}{r} \frac{d}{d r}\left(r \frac{d i Z}{d r}\right)-F(r) i Z+G(r) Y=-H_{2}(r)
$$

and further, introducing $X=i Z$ means equations (41) and (43) become

$$
\begin{aligned}
& \frac{A}{r} \frac{d}{d r}\left(r \frac{d Y}{d r}\right)-F(r) Y+G(r) X=H_{1}(r) \\
& \frac{A}{r} \frac{d}{d r}\left(r \frac{d X}{d r}\right)-F(r) X+G(r) Y=-H_{2}(r)
\end{aligned}
$$

The result of these manipulations is to effectively remove the imaginary part of the solution and so the equations can be treated as two coupled differential equations in real variables. 


\subsection{The Finite Difference Solution}

As mentioned above, equations (21), (29), (30), (23) and (24) must be solved numerically. To achieve this aim, a one dimensional finite difference scheme is utilised. Since there are rapidly changing potentials near the ischaemic border, the underlying grid is required to have a high concentration of nodes in this region, so a non-uniform grid is introduced. The grid is created using a technique for adjusting integration points in the approximate evaluation of singular integrals [16] which concentrates nodes at the singularity. This technique staggers the finite difference grid at different points depending on the position of the ischaemic boundary.

To use this non-uniform grid, divide the interval from $r=r_{a}$ to $r=r_{b}$ into $N$ subintervals of differing lengths, as described above. The nodes are then $r_{0}=r_{a}, r_{1}, \ldots r_{N-1}, r_{N}=r_{b}$, with the subinterval length $\Delta r_{j}=r_{j}-r_{j-1}$. For a function $y(r)$ defined on this grid, $y^{j}=y\left(r_{j}\right)$, and applying Taylor series expansions gives the approximations

$$
\left.\frac{d^{2} y}{d r^{2}}\right|_{r=r_{j}} \approx \frac{2\left(\Delta r_{j+1} y^{j-1}-\left(\Delta r_{j}+\Delta r_{j+1}\right) y^{j}+\Delta r_{j} y^{j+1}\right)}{\Delta r_{j} \Delta r_{j+1}\left(\Delta r_{j}+\Delta r_{j+1}\right)}
$$

and

$$
\left.\frac{d y}{d r}\right|_{r=r_{j}} \approx \frac{y^{j+1}-y^{j-1}}{\Delta r_{j}+\Delta r_{j+1}}
$$

for $j=1, \ldots, N-1$.

Application of these approximations to equations (21), (29) and (30) yields a tridiagonal system of linear equations of size $N+1$ which can be solved for the unknowns $C_{0 l}^{e}(r), C_{k 0}^{e}(r)$ and $D_{k 0}^{e}(r)$ after the system of equations has been completed by applying the appropriate boundary conditions. Using these approximations in equations (44) and (45) yields a pentadiagonal system of equations of size $2 N+2$. Solving this system gives the unknown functions $C_{k l}^{e}(r)$ and $D_{k l}^{e}(r)$, again after application of the appropriate boundary conditions. For the calculations presented below, $N$ was set to 200 . 


\subsection{Transformations of $\phi_{m}(z, \theta, r)$}

Recall from equation (6) that $\phi_{m}(z, \theta, r)$ is the product of three functions of the single variables $z, \theta$ and $r$. Firstly, the Fourier coefficients in $\theta$ of $\phi_{m}(z, \theta, r)$ are given by

$$
\begin{aligned}
A_{k}^{m}(r, z) & =\frac{1}{\pi} \int_{-\pi}^{\pi} \Psi\left(r+r_{a}\right) \Psi(z) \Psi(\theta) \cos k \theta d \theta \\
& =\frac{1}{\pi} \frac{2 \Psi\left(r+r_{a}\right) \Psi(z)}{1-e^{-a_{\theta} / \lambda_{\theta}}} \frac{1}{\lambda_{\theta}\left(\frac{1}{\lambda_{\theta}^{2}}+k^{2}\right)}\left\{(-1)^{k+1} e^{-\pi / \lambda_{\theta}} \sinh \frac{a_{\theta}}{\lambda_{\theta}}+\frac{\sin k a_{\theta}}{k \lambda_{\theta}}\right\}
\end{aligned}
$$

for $k=1,2, \ldots$ and

$$
A_{0}^{m}(r, z)=\frac{1}{2 \pi} \frac{2 \Psi\left(r+r_{a}\right) \Psi(z)}{1-e^{-a_{\theta} / \lambda_{\theta}}}\left\{a_{\theta}-\lambda_{\theta} \sinh \frac{a_{\theta}}{\lambda_{\theta}} e^{-\pi / \lambda_{\theta}}\right\}
$$

Note that $B_{k}^{m}(r, z)=0$ as $\Psi(\theta)$ is even in $\theta$, and hence $D_{k l}^{m}(r)=0$, as mentioned previously.

The next step is to take the Fourier transforms of equations (48) and (49) to determine the following quantities:

$$
\begin{aligned}
C_{k l}^{m}(r)= & \frac{\Psi\left(r+r_{a}\right)}{\pi} \frac{\sin 2 \pi l a_{z}}{1-e^{-a_{z} / \lambda_{z}}} \frac{1}{\pi l\left(1+4 \pi^{2} l^{2} \lambda_{z}^{2}\right)} \frac{2}{1-e^{-a_{\theta} / \lambda_{\theta}}} \frac{1}{\lambda_{\theta}\left(\frac{1}{\lambda_{\theta}^{2}}+k^{2}\right)} \\
& \times\left\{(-1)^{k+1} e^{-\pi / \lambda_{\theta}} \sinh \frac{a_{\theta}}{\lambda_{\theta}}+\frac{\sin k a_{\theta}}{k \lambda_{\theta}}\right\} \\
C_{k 0}^{m}(r)= & \frac{\Psi\left(r+r_{a}\right)}{\pi} \frac{2 a_{z}}{1-e^{-a_{z} / \lambda_{z}}} \frac{2}{1-e^{-a_{\theta} / \lambda_{\theta}}} \frac{1}{\lambda_{\theta}\left(\frac{1}{\lambda_{\theta}^{2}}+k^{2}\right)}\left\{(-1)^{k+1} e^{-\pi / \lambda_{\theta}} \sinh \frac{a_{\theta}}{\lambda_{\theta}}+\frac{\sin k a_{\theta}}{k \lambda_{\theta}}\right\} \\
C_{0 l}^{m}(r)= & \frac{\Psi\left(r+r_{a}\right)}{2 \pi} \frac{\sin 2 \pi l a_{z}}{1-e^{-a_{z} / \lambda_{z}}} \frac{1}{\pi l\left(1+4 \pi^{2} l^{2} \lambda_{z}^{2}\right)} \frac{2}{1-e^{-a_{\theta} / \lambda_{\theta}}}\left\{a_{\theta}-\lambda_{\theta} \sinh \frac{a_{\theta}}{\lambda_{\theta}} e^{-\pi / \lambda_{\theta}}\right\} \\
C_{00}^{m}(r)= & \frac{\Psi\left(r+r_{a}\right)}{2 \pi} \frac{2 a_{z}}{1-e^{-a_{z} / \lambda_{z}}} \frac{2}{1-e^{-a_{\theta} / \lambda_{\theta}}}\left\{a_{\theta}-\lambda_{\theta} \sinh \frac{a_{\theta}}{\lambda_{\theta}} e^{-\pi / \lambda_{\theta}}\right\}
\end{aligned}
$$

\subsection{Determining the Final Solution}

It is now possible to determine the value of $\phi_{e}$ at any point in the tissue and $\phi_{b}$ anywhere in the blood. Firstly, to determine $\phi_{e}$, for any mode number $k$ numerically solve the ordinary differential equations (23) and (24) for the Fourier transforms $C_{k l}^{e}(r)$ and $D_{k l}^{e}(r)$ for $l>0$ using the Numerical Recipes routines bandec and banbks [17], then determine $C_{k 0}^{e}(r)$ and $D_{k 0}^{e}(r)$ from equations (29) and (30) using the Numerical Recipes routine tridag. These Fourier transforms 
are then inverted using the discrete fast Fourier transform routine inft from Numerical Recipes at each value of $r$ on the one dimensional finite difference grid. The result of this inversion process is an array of values of $A_{k}^{e}(r, z)$ and $B_{k}^{e}(r, z)$. These coefficients, $A_{k}^{e}(r, z)$ and $B_{k}^{e}(r, z)$, are then used to calculate the potential $\phi_{e}$ through the original Fourier series representation of the solution, equation (12), at any angle $\theta$. The values of the coefficients for $k=0$ and $l>0$ are also evaluated from equation (21) using the tridag routine and inverted using the fast Fourier transform routine inft.

Note that inversion of the Fourier transforms in this fashion changes the boundary conditions in the $z$ direction. The computation domain is no longer infinite in the $z$ direction, but of finite extent, say from $-z_{0}$ to $z_{0}$ with the coefficients $A_{k}^{e}(r, z)$ and $B_{k}^{e}(r, z)$ defined at the grid nodes

in the $r$ direction and at equally spaced points a distance of $\frac{2 z_{0}}{2^{n}}$ apart in the $z$ direction. The boundary conditions have changed to the insulation type and care must be taken to ensure that the computation domain is large enough so that the original boundary conditions are not violated.

The same process is used to determine the potential distribution in the blood, $\phi_{b}$, except that the Fourier transforms can be obtained analytically, once the value of the corresponding Fourier transform of the tissue potential has been determined at $r=r_{a}$, the blood-tissue interface.

\section{Results}

The model described above will be used to compare the differences between a cylindrical model of the left ventricle and a previously published slab model of myocardial tissue $[3,6]$. Points of comparison will, firstly, be the effect of increasing the degree of subendocardial ischaemia [3] (using conductivity data from Clerc [5]) and, secondly, considering the effect of different 
conductivity data for a fixed sized ischaemic region [6].

As mentioned previously, the geometry of the model is described in Figure 1 where the inner cylinder has a radius of $2 \mathrm{~cm}\left(\right.$ ie $r_{a}=2$ ) and the outer cylinder has radius of $3 \mathrm{~cm}$ (ie $r_{b}=3$ ). For these particular simulations, the ischaemic region extends from $z=-2 \mathrm{~cm}$ to $z=2 \mathrm{~cm}$ (ie $a_{z}=2.0 \mathrm{~cm}$ ) and from $\theta=-\frac{\pi}{4}$ to $\theta=\frac{\pi}{4}$ (ie $a_{\theta}=\frac{\pi}{4}$ ). These values are chosen to maintain a similar ratio of ischaemic region size to domain size as for the slab model. For the first set of simulations, the degree of subendocardial ischaemia (that is $\left.\left(a_{r}-r_{a}\right) /\left(r_{b}-r_{a}\right) \times 100\right)$ was varied, taking values of $10 \%, 30 \%, 50 \%, 70 \%, 90 \%$ and full thickness, whereas for the second set of simulations it was fixed at $70 \%$ (ie $a_{r}=0.7 \mathrm{~cm}$ ). In all cases, a sharp interface between normal and ischaemic tissue was assumed with $\lambda_{\theta}=\lambda_{r}=\lambda_{z}=0.01$. Finally, the blood conductivity was set at $0.0067 \mathrm{~S} / \mathrm{cm}$.

For comparisons with previous simulations $[3,6]$, the fibre direction on the epicardial surface was aligned with the positive $z$-axis and allowed to rotate $120^{\circ}$ counter-clockwise to the endocardium. In terms of equation (3), $\psi_{0}=0^{\circ}$ and $\psi=120^{\circ}$.

\subsection{The Effect of Increasing Subendocardial Ischaemia}

Figure 2 shows the potential distributions on the outside surface of the cylinder as the degree of subendocardial ischaemia is increased. For the purposes of displaying the results, the cylinder has been cut along the $z$ direction, opposite the ischaemic region and laid out flat. Positive potentials are indicated with solid lines, negative potentials with dashed lines and zero potential is indicated by the thick solid line. The square in the centre of each panel is the projection of the ischaemic border onto the epicardial surface.

Increasing the degree of subendocardial ischaemia in the cylindrical model exhibits the same qualitative behaviour as observed in the slab model [3]. At 10\% ischaemia, there is a single valley of negative potential above the region of ischaemia which splits into three valleys at 
$30 \%$, one above the ischaemic region and two above the ischaemic boundary in the $\theta$ direction. By $50 \%$ ischaemia, areas of positive potentials arise as ridges between the valleys, with the central valley almost disappearing by $70 \%$. At $90 \%$ ischaemia, there is a region of positive potential above the ischaemia region, with valleys on both sides in the $\theta$ direction. Finally, at full thickness ischaemia, there is a region of positive potential above the region of ischaemia, surrounded on all four sides by regions of negative potential.

Generally, as the degree of ischaemia increases, the potential gradients above the ischaemic border increase and these are generally directed in the $\theta$ direction. At full thickness ischaemia there are also reasonably large potential gradients in the $z$ direction.

\subsection{The Effect of Differing Bidomain Conductivity Values}

Many experimental measurements of bidomain conductivities have been published (see [18]), with the most cited values obtained by Clerc [5], Roberts et al [19] and Roberts and Scher [20]. These values are summarised in Table 1. Figure 3 shows the epicardial potential distributions obtained using these three sets of conductivity values, as well a set based on the approximation of equal anisotropy ratios, all with $70 \%$ ischaemia. The format of the panels is the same as for Figure 2.

Simulations using the data of Clerc and Roberts et al, yield potential distributions which are qualitatively the same, with the data of Roberts et al giving the higher positive potentials and the lower negative potentials. The consequence of this is that larger potential gradients exist above the ischaemia border.

In contrast, the conductivity data of Roberts and Scher [20] yields a potential distribution which contains three valleys of negative potential, with only minimal positive potential on the opposite side of the ventricle. However, there are still large potential gradients above the ischaemic border in the $\theta$ direction. Also, using this conductivity data generates potentials 
which are more negative than with the previous two data sets.

Finally, the lower right panel of Figure 3, is based on the often used approximation in modelling bioelectrical phenomena of equal anisotropy ratios. The conductivity data used here is based on the data of Clerc [5] with $\sigma_{t}^{i}=\sigma_{l}^{i} \sigma_{t}^{e} / \sigma_{t}^{e}=0.0066 \mathrm{~S} / \mathrm{cm}$ using the data in Table 1. Here the high potential gradients above the ischaemic boundary have vanished, yet the minimum potential (still above the ischaemic regions) is more negative and the maximum potential is less positive than when the true values of Clerc are used (upper left panel).

\section{Discussion}

A similar model, based on a slab geometry, has been published previously [3] and it is interesting to compare the results of the simulations presented here with those from the slab geometry.

To begin with, consider the effect of changing the degree of ischaemia. A comparison of the two simulations, Figure 2 and Figure 4 in [3], shows a distinct similarity in the morphology of the potential distributions as the degree of ischaemia increases. However, there are a few minor differences. Firstly, the presence of the distinct valleys in the cylindrical model appears at about $30 \%$ ischaemia, whereas, it is not apparent until about $40 \%$ ischaemia in the slab model. Secondly, at 50\% ischaemia, in the cylindrical model, Figure 2(c), distinct regions of positive potential are observed, yet none are apparent until after $50 \%$ ischaemia in the slab model. At $70 \%$ ischaemia, there is no marked region of negativity above the region of subendocardial ischaemia in the cylindrical model, as is present in the slab model. However, the similarity is greatest at $90 \%$ ischaemia, with both models indicating an outline of the region of subendocardial ischaemia. Finally, at full thickness ischaemia, the patterns are again similar, except that the regions of negativity adjacent to the ischaemic regions are oriented in different directions. This difference is probably due to the way in which the fibre rotation differs between 
the two geometries, and also the periodicity of the domain in the $\theta$ direction.

Another point of comparison is the magnitude of the potentials. Table 2 gives a summary of the maximum and minimum potentials at each degree of ischaemia considered in Figure 2 for both the slab and the cylindrical geometries. Firstly, it can be seen that the magnitudes of the potentials in both models are of similar size, yet in the cylindrical model they are slightly greater than for the slab model. However, for both models, the ranges at each degree of ischaemia are very similar. The positive shift in the potentials in the cylindrical model is probably due to the smaller blood mass being available as a sink for the potential.

Now, consider the effect different conductivity values have on the epicardial potential distributions. Here, compare panels (a), (b) and (c) in Figure 3 with panels (c) in Figures 1, 2 and 3 in [6]. As above, the morphology of the potential distributions is very similar between the two geometries. The main difference occurs with the data of Roberts et al [19]; here for the slab geometry the positive peaks above the ischaemic region are isolated, whereas in the cylindrical geometry the regions of positivity extend all the way to the boundary. Again, as above, the potentials in the cylindrical model are higher than in the slab model. Even in the cylindrical model, there is a marked difference in the morphology and magnitudes of the epicardial potentials, again indicating the high sensitivity to the conductivity values.

In the case of equal anisotropy ratios, Figure 3(d), it is interesting to observe that considerable detail is lost in the potential distribution. It is also worth noting that using equal anisotropy ratios based on the data of Roberts et al. [19] and Roberts and Scher [20] yield potential distributions which are almost identical in shape (that is the zero lines and the maxima and minima are in the same place), except that the magnitudes of the potentials cover a greater range. It can then be argued from these observations that the approximation of assuming equal anisotropy ratios hides considerable detail in the epicardial potential distribution, which could in turn lead to incorrect interpretation of the simulated distributions. 
The results presented here can also be compared with previously published experimental data $[21,22,14]$. A detailed discussion of the comparison between experimental and modelled epicardial potential distributions has been published previously for the slab geometry [3]. Briefly, this mentioned that the model would predict the region of subendocardial ischaemia, which is not suggested by the experimental observations. In the cylindrical model presented here, this same conclusion is reached: the model does predict the location of the region of subendocardial ischaemia, although perhaps not as strongly as that suggested via the slab model, especially at the mid range levels of subendocardial ischaemia.

Secondly, although the potentials in the cylindrical model are somewhat higher than those in the slab model, they are still not close to the measured values. Again, possible reasons for this are the mathematical approximations necessary to render the problem suitable for the solution method proposed. Here, there is only one infinite dimension instead of three as in the slab geometry case. Hopefully, a more anatomically correct model of the left ventricle would produce more accurate values for the potentials.

Finally, the simplified shape of the ischaemic region may also account for differences between the model and experimental observations. It is probably a great simplification to assume that the $\theta$ and $z$ boundaries can be represented by flat surfaces as these boundary are more than likely irregular. Such irregularity could affect the local currents along the boundaries, which would in turn affect the epicardial potential distribution. It is also unlikely that the ischaemic region has a flat top. Assuming a non-uniform radial boundary would also induce different local currents, which would also affect the epicardial potential distribution.

On the other hand, the model does produce the high potential gradients in the vicinity of the ischaemic boundary as observed experimentally. It also follows the trend that the negative potentials become more negative as the subendocardial ischaemia approaches full thickness. 


\section{Conclusion}

This paper has presented a model for simulating subendocardial ischaemia in a cylindrical representation of the left ventricle. A detailed discussion of the modelling assumptions and the solution methods has been presented. The aim of presenting such detail is to establish analytic techniques (keeping the numerical aspects to a minimum) to model such physical phenomena in simple geometries which, in turn, can be used as test cases for more complex fully numerical methods for handling more complicated anatomically correct models.

Comparison between the results obtained in the cylindrical model presented here and a previously published slab model show that the morphologies of the epicardial potential distributions are similar, with the cylindrical geometry producing slightly higher values for the potentials. One main difference that arises is that, in the cylindrical geometry, positive potentials are observed at lower values of subendocardial ischaemia.

Finally, comparison of the model results with experimental observations has shown that the model can predict many of the features observed (large potential gradients, a deepening of the negative potentials with increasing subendocardial ischaemia) but not others (the fact that the model predicts the location of the region of subendocardial ischaemia, yet the experiments do not).

\section{References}

[1] C. C. Wolferth, S. Bettet, M. M. Livezey, and F. Murphy Negative displacement of the RS-T segment in the electrocardiogram and its relationships to positive displacement: an experimental study American Heart Journal, 29:220-244, 1945.

[2] H. Toyoshima, A. Ekmekci, E. Flamm, Y. Mizuno, T. Nagaya, R. Nakayama, K. Yamada, 
and M. Prinzmetal Angina pectoris VII. The nature of ST depression in acute myocardial ischaemia American Journal of Cardiology, 13:498-509, 1964.

[3] P. R. Johnston, D. Kilpatrick, and C. Y. Li The importance of anisotropy in modelling ST segment shift in subendocardial ischaemia IEEE Transactions on Biomedical Engineering, 48(12):1366-1376, December 2001.

[4] O. H. Schmitt Biological information processing using the concept of interpenetrating domains In K. N. Leibovic, editor, Information Processing in the Nervous System, chapter 18, pages 325-331 Springer-Verlag, New York, 1969.

[5] L. Clerc Directional differences of impulse spread in trabecular muscle from mammalian heart Journal of Physiology, 255:335-346, 1976.

[6] P. R. Johnston and D. Kilpatrick The effect of conductivity values on ST segment shift in subendocardial ischaemia IEEE Transaction on Biomedical Engineering, 50(2):150-158, February 2003.

[7] L. Tung A Bi-domain model for describing ischaemic myocardial $\mathrm{D}-\mathrm{C}$ potentials $\mathrm{PhD}$ thesis, Massachusetts Institute of Technology, June 1978.

[8] W. T. Miller and D. B. Geselowitz Simulation studies of the electrocardiogram: I the normal heart Circulation Research, 43:301-315, 1978.

[9] C. Henriquez Simulating the electrical behaviour of cardiac tissue using the bidomain model Critical Reviews in Biomedical Engineering, 21(1):1-77, 1993.

[10] B. Taccardi, E. Macchi, R. L. Lux, P. R. Ershler, S. Spaggiari, S. Baruffi, and Y. Vyhmeister Effect of myocardial fiber direction on epicardial potentials Circulation, 90:3076-3090, 1994. 
[11] D. D. Streeter Gross morphology and fiber geometry of the heart In R. M. Berne, editor, Handbook of Physiology, Vol 1, chapter 2: The Cardiovascular System, pages 61-112 Williams and Williams, Baltimore, MD, 1979.

[12] P. Colli-Franzone and L. Guerri Spreading of excitation in 3-D models of the anisotropic cardiac tissue i: Validation of the eikonal model Mathematical Biosciences, 113:145-209, 1993.

[13] I. J. Legrice, P. J. Hunter, and B. H. Smail Laminar structure of the heart: a mathematical model American Journal of Physiology, 272:H2466-H2476, 1997.

[14] D. Li, C. Y. Li, A. C. Yong, and D. Kilpatrick Source of electrocardiographic ST changes in subendocardial ischemia Circulation Research, 82:957-970, 1998.

[15] B. J. Roth A comparison of two boundary conditions used with the bidomain model of cardiac tissue Annals of Biomedical Engineering, 19:669-678, 1991.

[16] G. Monegato and I. H. Sloan Numerical solution of the generalised airfoil equation for an airfoil with a flap SIAM Journal of Numerical Analysis, 34(6):2288-2305, 1997.

[17] W. H. Press, B. P. Flannery, S. A. Teukolsky, and W. T. Vetterling Numerical Recipes, The Art of Scientific Computing Cambridge University Press, Cambridge, 2nd edition, 1992.

[18] B. J. Roth Electrical conductivity values used with the bidomain model of cardiac tissue IEEE Transactions on Biomedical Engineering, 44(4):326-328, April 1997.

[19] D. E. Roberts, L. T. Hersh, and A. M. Scher Influence of cardiac fiber orientation on wavefront voltage, conduction velocity and tissue resistivity in the dog Circ. Res., 44:701$712,1979$. 
[20] D. E. Roberts and A. M. Scher Effects of tissue anisotropy on extracellular potential fields in canine myocardium in situ Circ. Res., 50:342-351, 1982.

[21] R. A. Guyton, J. H. McClethan, G. E. Newman, and L. L. Michaelis Significance of subendocardial S-T segment elevation caused by coronary stenosis in the dog American Journal of Cardiology, 40:373-380, 1977.

[22] G. T. Smith, G. G. Geary, W. Blanchard, T. H. Roelofs, W. Ruf, and J. J. McNamara An electrocardiographic model of myocardial ischaemic injury Journal of Electrocardiology, 16(3):223-234, 1983. 


\section{Table Captions}

Table 1: Measured bidomain conductivities $(\mathrm{S} / \mathrm{cm})$.

Table 2: Minimum and maximum epicardial potentials (in $\mathrm{mV}$ ) for the two different geometries.

\section{Figure Captions}

Figure 1: Cross-section of the cylindrical model at $z=0$.

Figure 2: Epicardial surface potential distributions (in $\mathrm{mV}$ ) for different degrees of subendocardial ischaemia using conductivity data from Clerc [5]. For the purposes of displaying the results, the cylinder has been cut along the $z$ direction, opposite the ischaemic region and laid out flat. The fibres on the epicardial surface are directed parallel to the $z$-axis. Positive potentials are indicated with solid lines, negative potentials with dashed lines and zero potential is indicated by the thick solid line. The square in the centre of each panel is the projection of the ischaemic border onto the epicardial surface.

Figure 3: Epicardial surface potential distributions (in $\mathrm{mV}$ ) for different sets of bidomain conductivity values at $70 \%$ ischaemia. The format for each panel is the same as in Figure 2. 


\begin{tabular}{|c|c|c|c|}
\hline \multirow{2}{*}{} & \multicolumn{3}{|c|}{ Bidomain Conductivities (S/cm) } \\
\cline { 2 - 4 } & Clerc [5] & Roberts et al. [19] & Roberts \& Scher [20] \\
\hline$\sigma_{l}^{i}$ & 0.0017 & 0.0028 & 0.0034 \\
$\sigma_{t}^{i}$ & 0.00019 & 0.00026 & 0.0006 \\
$\sigma_{l}^{e}$ & 0.0062 & 0.0022 & 0.0012 \\
$\sigma_{t}^{e}$ & 0.0024 & 0.0013 & 0.0008 \\
\hline
\end{tabular}

Table 1:

\begin{tabular}{|c|c|c|c|c|}
\hline \multirow{2}{*}{$\begin{array}{c}\text { Degree } \\
\text { of }\end{array}$} & \multicolumn{2}{|c|}{ Slab Geometry } & \multicolumn{2}{c|}{ Cylindrical Geometry } \\
\cline { 2 - 5 } Ischaemia (\%) & \multicolumn{2}{|c|}{ Potential (mV) } & \multicolumn{2}{c|}{ Potential (mV) } \\
\cline { 2 - 5 } & Minimum & Maximum & Minimum & Maximum \\
\hline 10 & -1.2 & 0 & -1.0 & 0.1 \\
30 & -0.9 & 0 & -0.7 & 0.06 \\
50 & -0.9 & 0.1 & -0.8 & 0.13 \\
70 & -1.3 & 0.3 & -1.2 & 0.6 \\
90 & -1.9 & 0.9 & -1.7 & 1.2 \\
Full Thickness & -2.1 & 4.4 & -1.9 & 4.6 \\
\hline
\end{tabular}

Table 2: 


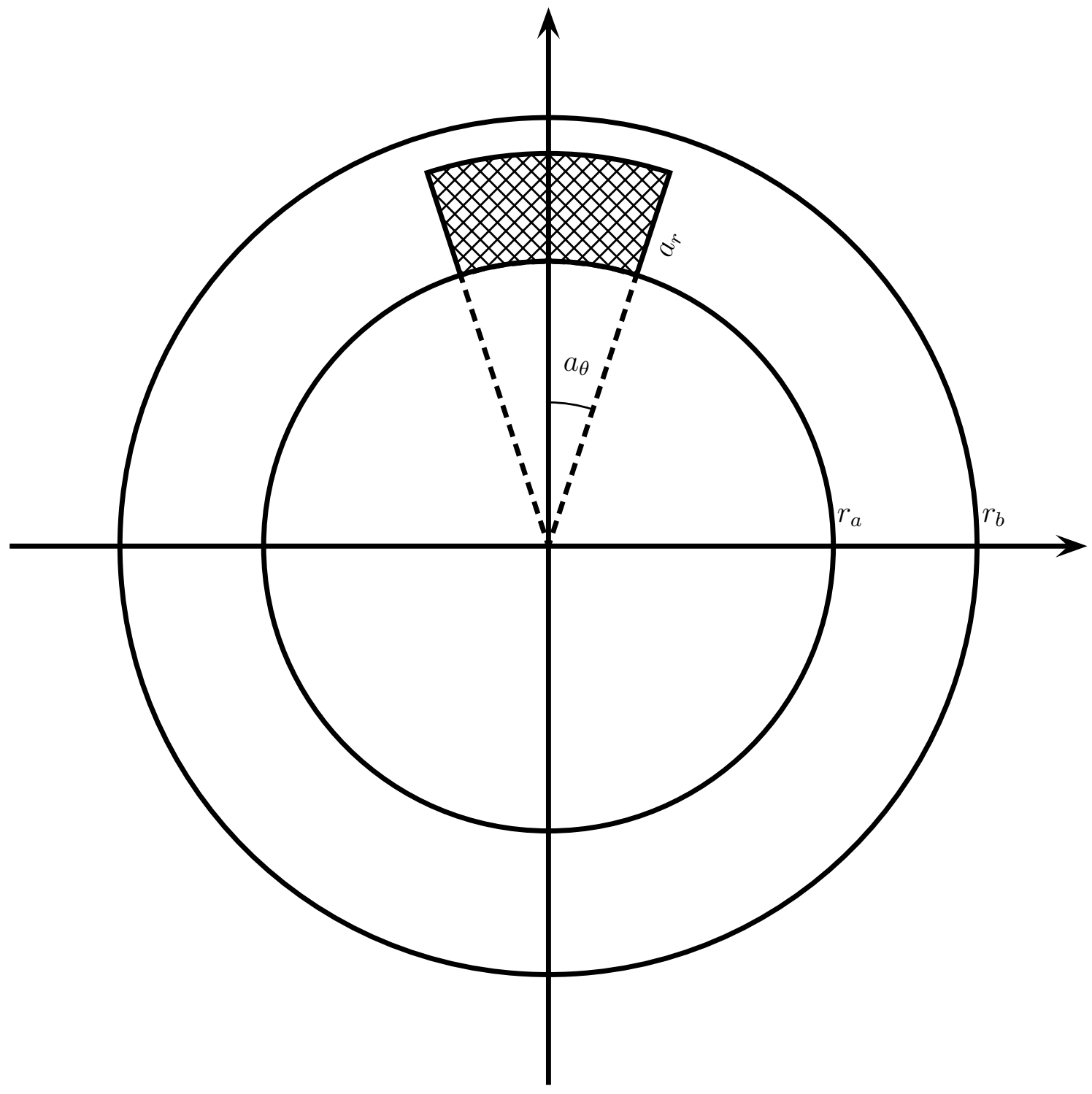

Figure 1: 
Epicardial Potential Distribution (mV)

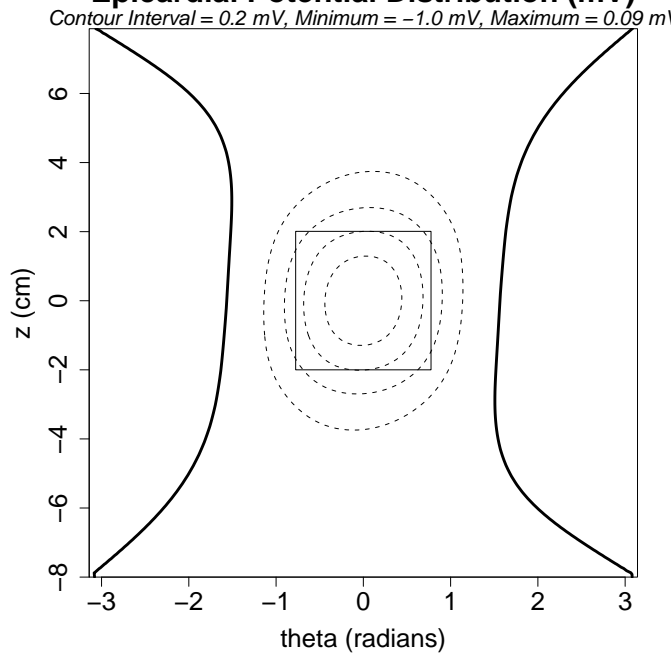

(a) $10 \%$ Ischaemia

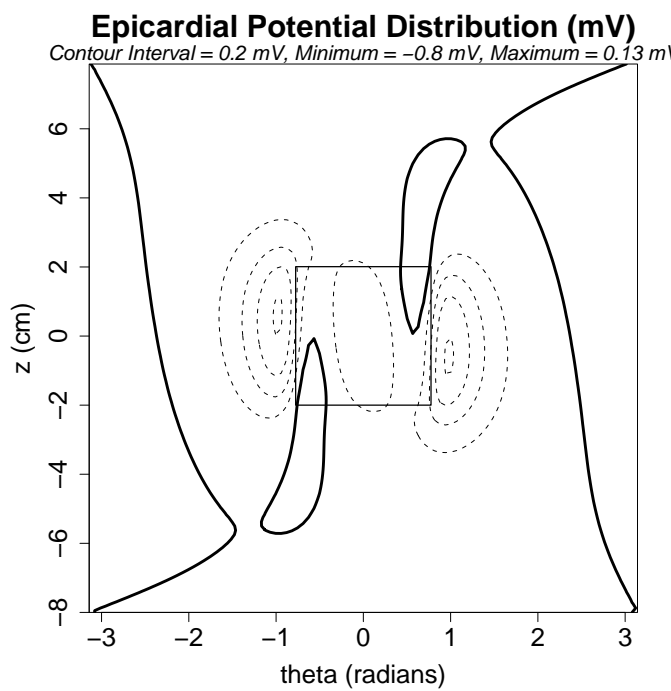

(c) $50 \%$ Ischaemia

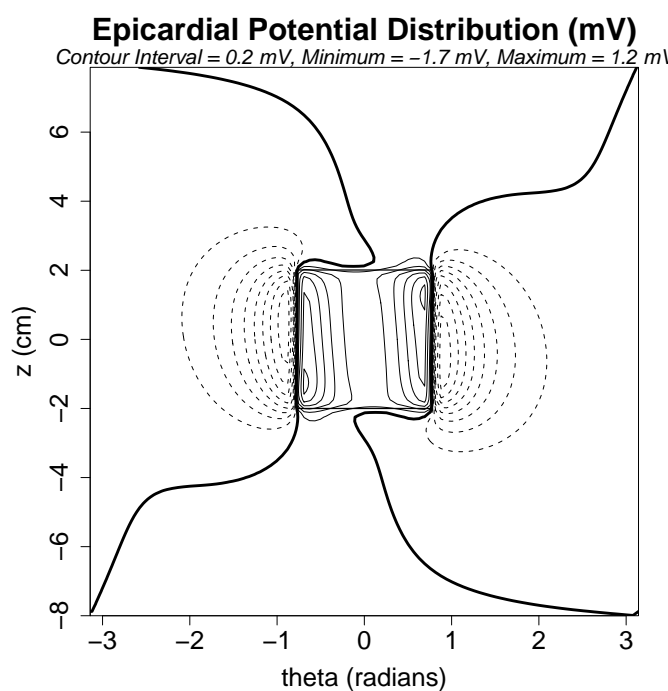

(e) $90 \%$ Ischaemia
Epicardial Potential Distribution (mV)

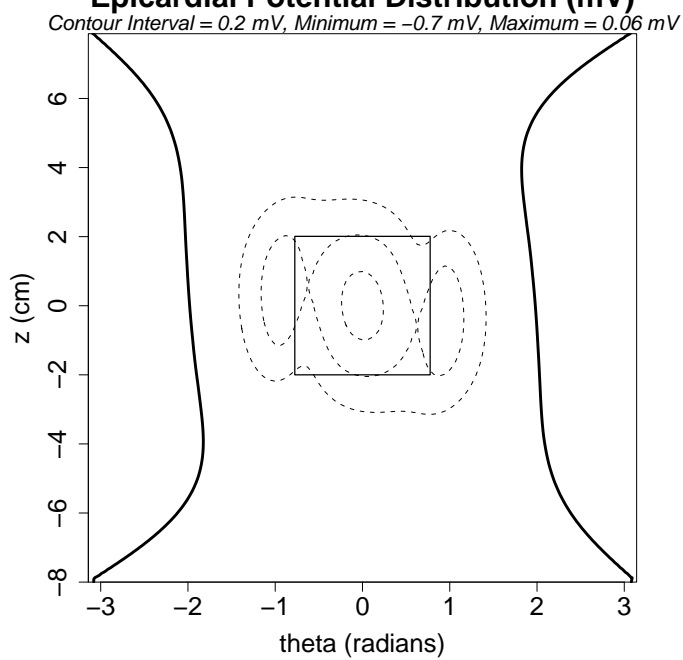

(b) $30 \%$ Ischaemia

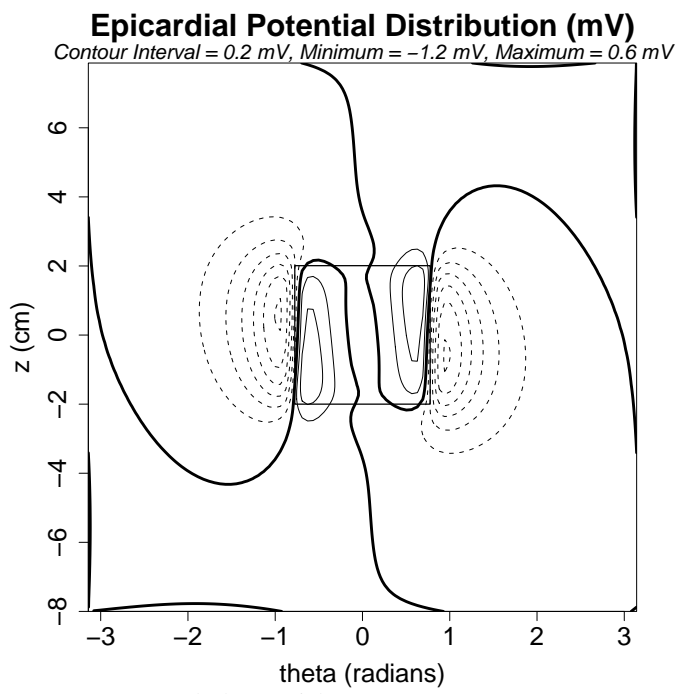

(d) $70 \%$ Ischaemia

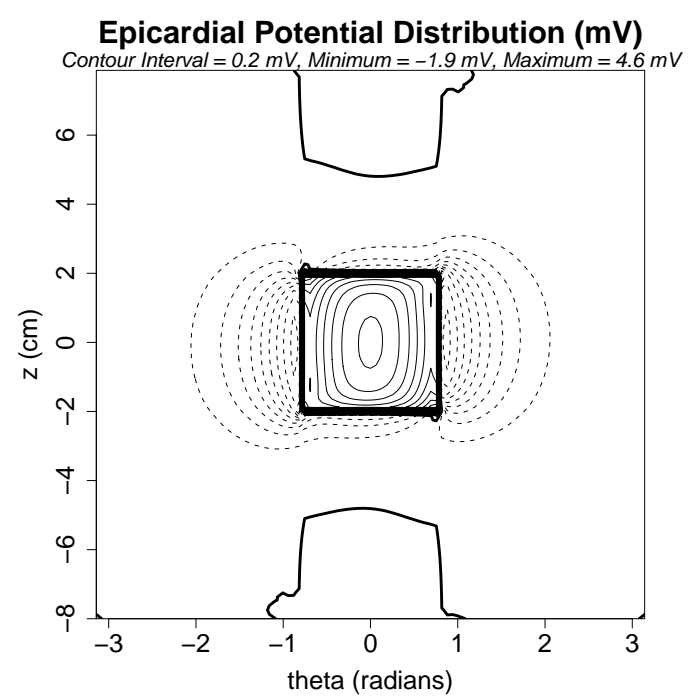

(f) Full Thickness Ischaemia

Figure 2: 


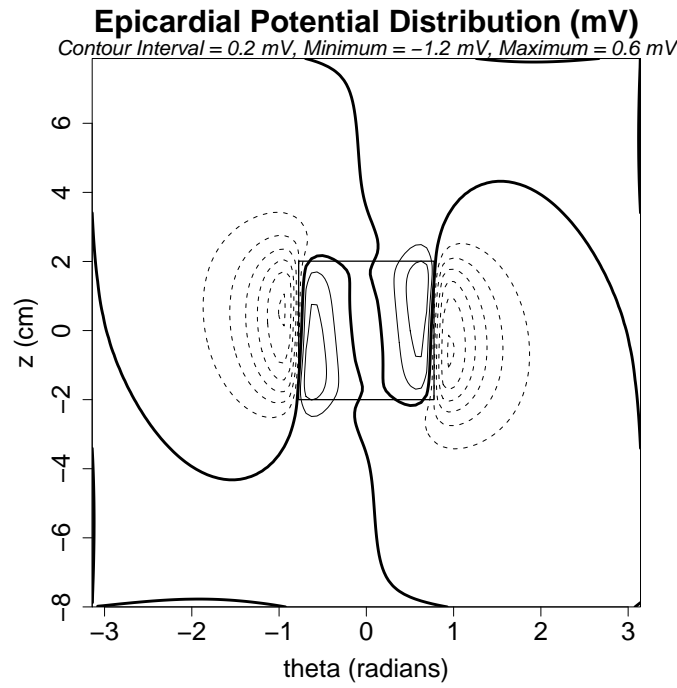

(a) Using Conductivity Data

from Clerc [5]

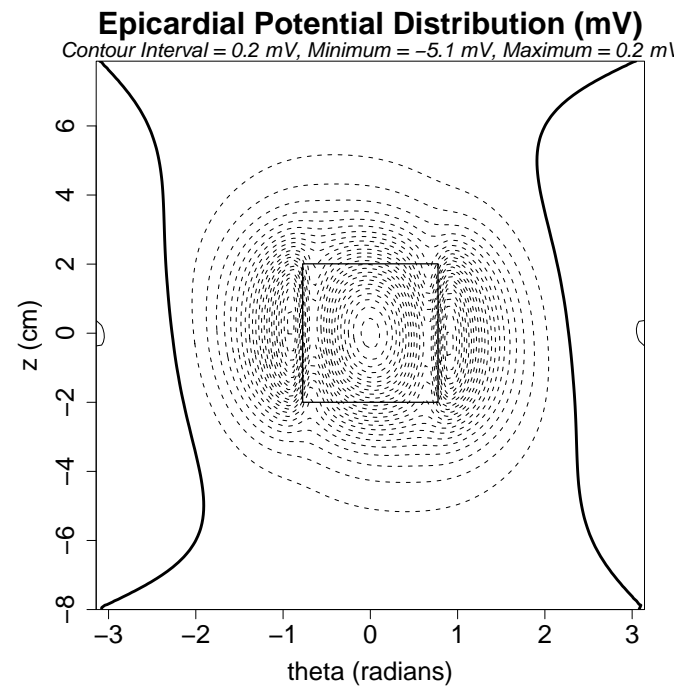

(c) Using Conductivity Data

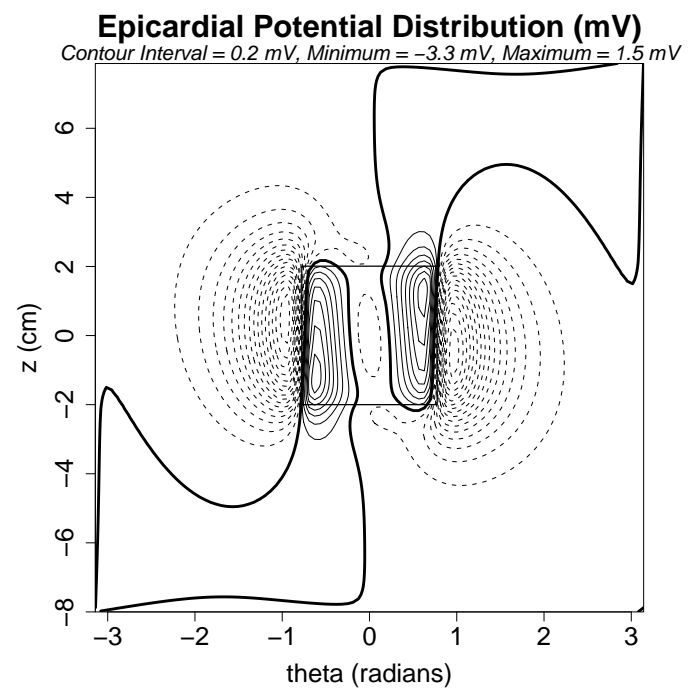

(b) Using Conductivity Data

from Roberts et al [19]

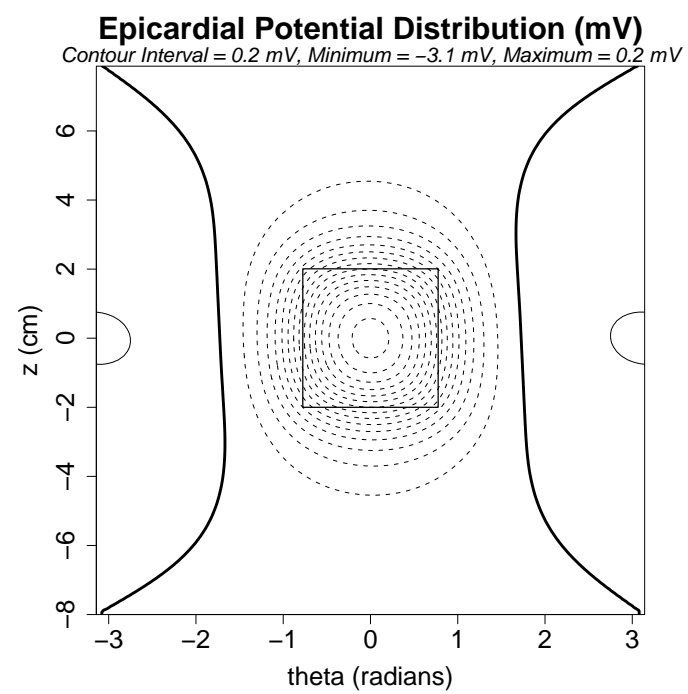

(d) Using Equal Anisotropy Ratios

from Roberts and Scher [20]

Figure 3: 